\title{
Intensive follow-up after liver resection for colorectal liver metastases: results of combined serial tumour marker estimations and computed tomography of the chest and abdomen - a prospective study
}

\author{
S Bhattacharjya', R Aggarwal' and BR Davidson*,I \\ 'Department of Surgery, Royal Free and University College School of Medicine, University College London, The Royal Free Hospital, Pond Street, London \\ NW3 2QG, UK
}

\begin{abstract}
The aim of the study was to prospectively evaluate an intensive follow-up programme using serial tumour marker estimations and contrast-enhanced computed tomography (CT) of the chest and abdomen in patients undergoing potentially curative resection of colorectal liver metastases. Seventy-six consecutive patients having undergone potentially curative resections of colorectal liver metastases in a single unit were followed up with a protocol of 3 monthly carcinoembryonic antigen and carbohydrate antigen 19-9 estimations and contrast-enhanced spiral CT of the chest, abdomen and pelvis for the first 2 years following surgery and 6 monthly thereafter. The median period of follow-up was 24 months (range 18-60). Recurrent tumour was classed as early if within 6 months of liver resection. Thirty-seven of the 76 patients (49\%) developed recurrence on follow-up. Nineteen recurrences were in the liver alone (5I\%), I 6 liver and extrahepatic (43\%) and two extrahepatic alone (6\%). Of the 19 patients with isolated liver recurrence, eight developed within 6 months of liver resection none of which were resectable. Of the II recurrences after 6 months, five (45\%) were resectable. Of the 37 recurrences, CT indicated recurrence despite normal tumour markers in 19 patients. Tumour markers suggested recurrence before imaging in 12 and concurrently with imaging in 6 . In the 12 patients who presented with elevated tumour markers before imaging, there was a median lag period of 3 months (range $\mid-2 I$ ) in recurrence being detected on further serial imaging. Seventeen patients who developed recurrence had normal tumour markers before initial resection of their liver metastases. Of these 17, 10 (58\%) had an elevation of tumour markers associated with recurrence. Over a median follow-up of 2 years following liver resection, the use of CT or tumour markers alone would have failed to demonstrate early recurrence in 12 and I 8 patients respectively. A combination of tumour markers and CT detected significantly more $(P<0.05)$ recurrence than either modality alone. Tumour markers and CT should be used in combination in the follow-up of patients with resected colorectal liver metatases, including patients whose markers are normal at the time of initial liver resection. British Journal of Cancer (2006) 95, 21 -26. doi:10.1038/sj.bjc.6603219 www.bjcancer.com
\end{abstract} (c) 2006 Cancer Research UK

Keywords: colorectal liver metastases; follow-up; tumour markers; imaging

The incidence of colorectal cancer in the Western World is approximately 50 per 100000 with a cumulative lifetime risk of approximately 5\% (Rudy and Zdon, 2000; Malafosse et al, 2001; McCormick et al, 2002). Liver metastases are one of the principal causes of death in these patients. About $25 \%$ of patients have liver metastases at the time of presentation and another $20 \%$ subsequently develop liver metastases (Norum et al, 1997; Lyass et al, 2001). Median survival of untreated colorectal liver metastases is approximately 9 months with few survivors beyond 2 years (Bengtsson et al, 1981; Fujimoto et al, 1985; Giacchi et al, 1988; Daly et al, 1989). The reported 5-year survival following surgical resection of liver metastases is between 35 and $40 \%$ (Scheele et al,

*Correspondence: Professor BR Davidson;

E-mail: b.davidson@medsch.ucl.ac.uk

Received 3 February 2006; revised 20 April 2006; accepted I 5 May 2006
1990; Ballantyne and Quin, 1993; Leow et al, 1997; Beard et al, 2000; Bolton and Fuhrman, 2000; Ruiz et al, 2000; Weber et al, 2000; Yamaguchi et al, 2000; Ruers and Bleichrodt, 2002).

A variety of imaging strategies have been suggested in the follow-up of patients after resection of colorectal liver metastases (Tartter et al, 1981; Fantini and DeCosse, 1990; Baulieu et al, 2001; Glover et al, 2002; Longo and Johnson, 2002). The presence of recurrent liver metastases was historically considered to have a poor prognosis and routine screening for patients having undergone resection of colorectal liver metastases was felt not to be justified by analysis of cost to benefit ratio (Biggs and Ballantyne, 1994; Beard et al, 2000). However, recent studies have shown a $35-$ $40 \%$ survival at 5 years following surgery for recurrent liver metastases (Tuttle et al, 1997; Yamamoto et al, 1999a; Muratore et al, 2001; Suzuki et al, 2001; Yamada et al, 2001a). These results reflect a better outcome with a more aggressive approach towards the treatment of colorectal liver metastases and careful follow-up 
of patients following resection of the liver metastases is therefore essential.

The main follow-up options for patients with resected colorectal liver metastases combine serial estimations of tumour markers with imaging by ultrasound, computed tomography (CT), magnetic resonance imaging or position emission tomography (PET) scan. Spiral CT has a high accuracy in the detection of colorectal liver metastases (Bhattacharjya et al, 2004). This prospective study presents the results of using serial tumour marker estimation in conjunction with contrast-enhanced CT of the chest, abdomen and pelvis in the follow-up of patients following liver resection for colorectal metastases.

\section{MATERIALS AND METHODS}

Prospectively collected data on 76 patients who had undergone potentially curative resection for colorectal liver metastases in a single centre over a 5-year period from January 1996 to December 2001 was reviewed. During this period 120 patients were referred for consideration of liver surgery for metastases following a curative resection of a colorectal cancer. Staging investigations over this time period included contrast CT of the chest, abdomen and pelvis, a dynamic gadolinium enhanced magnetic resonance of the liver and a CT arterial portography. At surgery these patients were further assessed with intra-operative ultrasound and bimanual palpation. The results of this staging algorithm have been previously described (Bhattacharjya et al, 2004).

Eighty-nine patients had resection of their liver metastases. Of these 13 were excluded from the current follow-up study owing to noncurative liver resections (margin positive $n=4$ ) or incomplete follow-up $(n=9)$. All 76 patients had tumour marker estimation before liver resection and at 6 weeks following surgery. The patients entered a follow-up protocol of measuring serum carcinoembryonic antigen (CEA) and carbohydrate antigen 19-9 (CA 19-9) plus contrast-enhanced spiral CT (CECT) of the chest and abdomen at 3 monthly intervals for the first 2 years and thereafter at 6 monthly intervals till 5 years. The imaging protocol for the contrast CT of the chest and abdomen during follow-up was the same as used for pre-operative staging and is outlined below. The follow-up imaging was carried out at the Royal Free Hospital. The minimum period of follow-up was 18 months, median 24 months.

\section{Technique of CECT of the chest}

Contrast-enhanced axial images of the chest were obtained on a GE HiSpeed spiral CT with $10 \mathrm{~mm}$ collimation at $10-15 \mathrm{~m} \mathrm{~s}^{-1}$ table speed $20 \mathrm{~s}$ following injection of $75-100 \mathrm{ml}$ Omnipaque 300 (Nycomed, Amersham Health, Little Chalfont, Bucks, UK) through a peripheral vein. Well-defined nonenhancing lesions of soft tissue density in the lung parenchyma were considered to be suspicious and were followed up by either fluoro-2-deoxy-D-glucose (FDG) PET scan or repeat CECT of the chest at 6 weeks. Lesions that showed uptake of FDG or had increased in size were classified as pulmonary metastases.

\section{Technique of CECT of the abdomen}

Portal phase images were obtained $70 \mathrm{~s}$ following injection of $75-$ $100 \mathrm{ml}$ Omnipaque 300 through a peripheral vein infused at a rate of $4 \mathrm{ml} / \mathrm{s}^{-1}$ on a GE HiSpeed spiral CT with $7-10 \mathrm{~mm}$ collimation at $10-15 \mathrm{~mm} \mathrm{~s}^{-1}$ table speed. The abdominal scans were performed from the level of the domes of the diaphragm to the symphysis pubis. Continguous 7- to-10-mm-thick axial images were reconstructed from the volumetric data. Well-defined nonenhancing water density lesions were considered to be benign. Isoor hypodense lesions with rim enhancement were considered to represent metastases. Consultant radiologists with an interest in hepatobiliary radiology reviewed the images. An ordinal category system was used to group the patients with liver metastases into solitary metastases $\mathrm{H} 1,2-5$ metastases $\mathrm{H} 2$ and $>5$ metastases $\mathrm{H} 3$.

Carcinoembryonic antigen and CA19-9 levels were assessed in the serum by immunoassay techniques performed on the Roche Analyser E170 (Roche Diagnostics, Lewes, East Sussex UK). Serum CEA levels greater than $5 \mathrm{ng} \mathrm{ml}^{-1}$ and CA 19-9 levels greater than $37 \mathrm{U} \mathrm{ml}^{-1}$ were considered abnormal. Tumour marker levels were correlated with findings on imaging and clinical follow-up of the patients. The effect of the following variables on disease-free survival was assessed: Duke's stage of bowel cancer, tumour marker levels, number of liver metastases and adjuvant chemotherapy for the primary colon cancer.

A cost analysis of the protocol based on costs for private investigations in an NHS hospital was performed. The cost of the follow-up surveillance programme was evaluated based on an estimated cost of a spiral CT at $£ 500.00$ and cost per CEA and CA19-9 estimation at $£ 5.00$ per test. The cost of clinician follow-up time was not analysed.

Statistical analysis was performed using SPSS version 10 Survival was assessed using Kaplan-Meier curves and compared by the log-rank test. $\chi^{2}$ and where appropriate Fishers exact test were used. Statistical significance was considered to be present on $P<0.05$.

\section{RESULTS}

Seventy-six consecutive patients who had undergone liver resection for colorectal liver metastases were analysed in this follow-up study. The median age was 60 years (range 29-77) with a male-tofemale ratio of $40: 36$. Fifty of the 76 patients presented with synchronous metastases, whereas 26 had metachronous metastases. Forty-two had a node negative and 34 a node-positive primary tumour. All primary resections had clear histological resection margins. Thirty patients had a solitary metastasis, 44 had $2-5$ and $2>5$ metastases (Table 1). Nineteen patients underwent a right or left hepatectomy, 13 an extended right or left hepatectomy and 38 a segmental $(n=20)$ or nonanatomical wedge hepatic resection $(n=18)$. Five patients had an extended hepatectomy plus wedge resection (Table 2).

Results of CT scans were available for all 76 patients and there were a total of eight scans per patient during the median 24 month follow-up (range of follow-up 18-60 months). Fifty-four patients

Table I Profile of patients undergoing liver resection for colorectal metastases

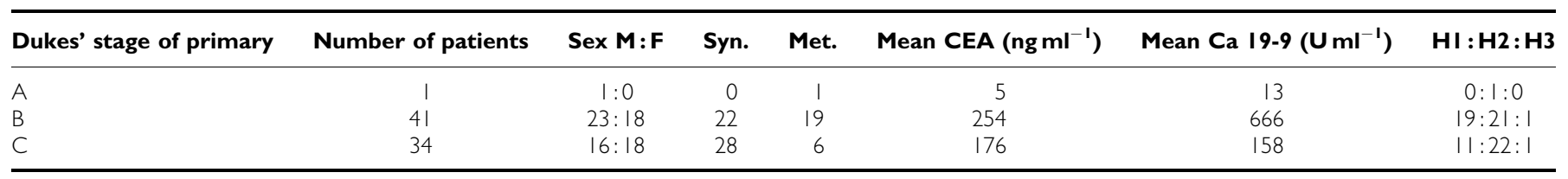

Syn., synchronous colorectal liver metastases; Met., Metachronous colorectal liver metastases; mean CEA, at time of initial liver resection (normal levels I - 5 ng ml ${ }^{-1}$ ); mean $\mathrm{CA}$ 19-9, At the time of initial liver resection (normal levels $17-37 \mathrm{U} \mathrm{ml}^{-1}$ ); $\mathrm{HI}$, solitary metastasis; $\mathrm{H2}, 2-5$ liver metastases; $\mathrm{H3}$, >5 liver metastases. 
Table 2 Initial liver resections performed

\begin{tabular}{|c|c|c|c|c|c|c|}
\hline $\begin{array}{l}\text { Dukes' } \\
\text { stage }\end{array}$ & R Hemihepatectomy & $\begin{array}{c}\text { Extended R } \\
\text { Hemihepatectomy }\end{array}$ & L Hemihepatectomy & $\begin{array}{c}\text { Extended L } \\
\text { Hemihepatectomy }\end{array}$ & $\begin{array}{l}\text { Segment/wedge } \\
\text { resection }\end{array}$ & $\begin{array}{c}\text { Ext. hemihepatectomy+ } \\
\text { wedge }\end{array}$ \\
\hline A & & & & & । & \\
\hline C & 5 & 4 & 3 & 2 & 18 & 2 \\
\hline Total & 15 & 12 & 4 & 2 & 38 & 5 \\
\hline
\end{tabular}

$L$, left, $R$, right.

Table 3 Sites of recurrence during follow-up of 76 patients with resected colorectal liver metastases

\begin{tabular}{lccc}
\hline Recurrence sites & Total no. & $\begin{array}{c}\text { Recurrence } \\
<\mathbf{6 / 1 2}\end{array}$ & $\begin{array}{c}\text { Recurrence } \\
>\mathbf{6 / 1 2}\end{array}$ \\
\hline Liver only & 19 & 8 & 11 \\
Liver+lungs & 13 & 5 & 8 \\
Liver+lymph nodes & 3 & 1 & 2 \\
Other & 2 & 1 & 1 \\
\hline
\end{tabular}

Recurrence $<6 / 12$, recurrence diagnosed within 6 months of liver resection; recurrence $>6 / 12$, recurrence diagnosed beyond 6 months of liver resection.

were followed up at our centre exclusively and eight results for tumour marker estimations were available for each of these patients during the follow-up period. Twenty-two patients were followed up jointly with their referring hospitals. In these 22 patients, the tumour marker estimation was performed locally and a median of 6 (range $4-8$ ) results was available for this group.

No recurrence was detected in 39 patients over the follow-up period. Thirty-seven patients had a recurrence. Fifteen presented with recurrence within 6 months of the liver resection and 22 more than 6 months following liver resection (Table 3). Nineteen had recurrence in the liver only, 13 liver and lungs, three liver and hilar and celiac lymph nodes and two had extrahepatic recurrence (Table 3). Recurrence following liver resection was not influenced by the Dukes stage of the primary bowel cancer, 20 of the 41 patients with a Dukes B cancer developed recurrence (49\%) and 17 of the 34 with a Dukes $C$ stage cancer (50\%). Of the 20 recurrences who had Dukes B cancers, six recurred within and 14 after 6 months of liver resection. Of the 17 Dukes $C$ recurrences nine recurred within 6 months and eight after 6 months. Delayed recurrence was more common in the Dukes' B group (14 out of 20, $70 \%$ ) than the Dukes' C group (eight out of 17, 47\%), although this was not statistically significant.

Of the patients with recurrent metastases, 15 underwent initial resection for solitary liver metastases (H1), $212-5$ metastases (H2) and $1>5$ metastases (H3). Nine patients had a right or left hepatectomy, seven an extended hepatectomy, 18 had a wedge resection and three an extended hepatectomy plus wedge resection for their liver metastases at initial presentation (Table 4). No association was observed in the type of resection performed or number of tumours ( $\mathrm{H}$ stage) and the timing of recurrence being detected.

Of the 76 patients included in this study, 65 had raised (secreting tumours) and 17 had normal (nonsecreting tumours) tumour markers before the first liver resection. In the 37 patients with recurrences, 12 (group 1) had raised tumour markers before CT evidence; six (group 2) had tumour markers correlating with CT evidence and 19 patients (group 3) had CT evidence of recurrence before elevation of tumour markers. In group 1, five patients had elevated tumour markers at the time of initial liver resection and had therefore, secreting tumours. In groups 2 and 3 , three and 12 patients had secreting tumours respectively (Table 5). In total, 20 of the 37 patients with recurrences had secreting tumours and in them elevated tumour markers were predictive of recurrence in eight $(40 \%)$. Seventeen patients did not have a secreting tumour and in $10(59 \%)$ elevated tumour markers on follow-up were predictive of recurrence. Raised tumour markers were the first indication of recurrent disease in 18 of 76 patients (24\%). Normal tumour marker levels with CT evidence of recurrence were noted in 19 of 76 patients $(25 \%)$. A protocol of using both tumour markers and CT in the postoperative period significantly improved the diagnostic accuracy and detected recurrent disease in 37 of 76 patients $(49 \%)(P<0.05)$.

Of the 12 patients with rise in tumour markers and normal CTs, CEA was elevated in eight and CA 19-9 in nine (both in 5). Of the six where tumour markers and imaging both suggested a recurrence five patients had an elevated CEA and five elevated CA 19-9. In the 12 patients with elevated tumour markers with normal CT, there was a median lag period of 3 months (range 1-21 months) before recurrence was detected on further imaging.

Nineteen patients had recurrent metastases in the liver alone. Eight $(42 \%)$ presented within 6 months of surgery. None of these had resectable disease on repeat staging. Eleven (58\%) presented after 6 months, of whom five $(45 \%)$ were resectable. Four of these patients underwent a further liver resection. One patient refused further surgery. One of the patients undergoing a repeat hepatic resection developed a further recurrence at 18 months. The other three patients have remained well and disease-free (median followup of 30 months (range 12-54 months)). Of the 53 patients who had received chemotherapy before the initial liver resection, 26 (49\%) developed recurrent disease (18 Dukes' B and 8 Dukes' C). Of the 23 patients who did not receive initial chemotherapy, 11 (49\%) developed recurrence.

The cost of the current protocol that identified five out of 76 patients who could have been potentially cured by repeat liver resection was $£ 310080.00$ over a 2-year period (cost per CT scan $=£ 500.00$; cost per CEA and CA19-9 estimation at $£ 5.00$ per test; total of $(76 \times 8) 608$ scans and blood tests $)$. Therefore, the cost per patient potentially cured per year was $£ 31000$. The cost of following up an individual patient by this protocol was $£ 2040.00$ per year.

\section{DISCUSSION}

Intensive follow-up programmes to screen for recurrent cancer should be designed to detect disease in a selected population where a therapeutic intervention altering ultimate prognosis and survival can be offered. Where this is not possible, these tests unnecessarily enhance patient anxiety and may reduce their overall quality of life. A selective approach is often required to justify the costs of screening, which has an unacceptable costbenefit ratio when applied universally (Beard et al, 2000; Gazelle et al, 2003).

The surveillance programme used in this study of patients who had undergone resection of colorectal liver metastases detected recurrent disease in $49 \%$ of patients. Of these recurrences, $51 \%$ 
Table 4 Patterns of recurrence

\begin{tabular}{|c|c|c|c|c|}
\hline Dukes' stage of primary & Number of patients & No recurrence & Recurrence $<6 / 12$ & Recurrence $>6 / 12$ \\
\hline A & 1 & 1 & 0 & 0 \\
\hline B & 41 & 21 & 6 & 14 \\
\hline C & 34 & 17 & 9 & 8 \\
\hline Number of metastases & No. of patients & No recurrence & Recurrence $<6 / 12$ & Recurrence $>6 / 12$ \\
\hline $\mathrm{HI}(<2)$ & 30 & 15 & 6 & 9 \\
\hline $\mathrm{H}_{2}(2-5)$ & 44 & 23 & 9 & 12 \\
\hline $\mathrm{H} 3(>5)$ & 2 & I & 0 & I \\
\hline
\end{tabular}

Liver resection

$\mathrm{R}$ hemihepatectomy

Ext. R hemihepatectomy

$L$ hemihepatectomy

Ext. $L$ hemihepatectomy

Segmental or wedge resection

Ext. hemihepatectomy+wedge
No. of patients

No recurrence

Recurrence $<6 / 12$

Recurrence $>6 / 12$

15
12
4
2
38
5

$\begin{array}{rrr}8 & 4 & 3 \\ 6 & 4 & 2 \\ 2 & 0 & 2 \\ 1 & 0 & 1 \\ 20 & 7 & 11 \\ 2 & 0 & 3\end{array}$

Recurrence $<6 / 12$, recurrence diagnosed within 6 months of liver resection; recurrence $>6 / 12$, recurrence diagnosed beyond 6 months of liver resection; HI, solitary liver metastasis; H2, 2 -5 liver metastases; H3, > 5 liver metastases.

Table 5 Results of surveillance

Surveillance result

CEA/CA 19-9 raised before CT evidence

CT evidence before raised CEA/CA 19-9

CT and CEA/CA 19-9 correlate evidence
CEA/CA $19-9$ elevated

Recurrence $<6 / 12$

Recurrence $>6 / 12$ before liver resection

$\begin{array}{rrr}5 & 7 & 5(2,3) \\ 8 & 11 & 12(6,6) \\ 2 & 4 & 3(2,1)\end{array}$

CEA, carcinoembryonic antigen $>5 \mathrm{ng} \mathrm{ml}^{-1}$; CA 19-9, carbohydrate antigen 19-9 >37 $\mathrm{mml}^{-1}$; CT, computed tomography; recurrence $<6 /$ 2, recurrence diagnosed within 6 months of liver resection; recurrence $>6 / 12$, recurrence diagnosed beyond 6 months of liver resection.

were in the liver alone, $43 \%$ liver and extrahepatic and $6 \%$ extrahepatic alone. This incidence and pattern of recurrence is similar to data previously published (Sugihara et al, 1993) and confirms that the majority of patients developing recurrence develop liver- alone disease, which could potentially be amenable to further liver surgery (Topal et al, 2003). Where surgery is not possible, alternate therapies like radiofrequency ablation may provide local tumour control (Gillams and Lees, 2005).

None of the patients with recurrent liver metastases detected within 6 months of their liver resection had resectable disease. These early recurrences may represent microscopic metastatic disease or occult metastases present at the time of liver resection that only becomes apparent under the trophic effects of liver regeneration. Local recurrence owing to incomplete resection of the liver metastases is unlikely as patients with histologically involved liver resection margins were not included in this followup study. The ability to detect occult disease is limited by the technical resolution of currently available imaging equipment. Intra-operative ultrasound is the most sensitive technique and all these patients had had an intra-operative ultrasound at the time of first liver resection (Machi et al, 1987; Agrawal et al, 2006). As none of these patients proved to be amenable to further liver resection they will represent a poor prognosis group in whom surveillance is less likely to be beneficial.

Of the patients who developed liver metastases after 6 months, a further curative resection was possible in $45 \%$. The reported 5 -year survival in patients undergoing repeat resections of their liver metastases is in the region of 35-40\% (Yamamoto et al, 1999a; Beard et al, 2000; Muratore et al, 2001; Suzuki et al, 2001; Yamada et al, 2001a; Gazelle et al, 2003) and therefore a follow-up aimed at detecting these resectable recurrences is useful and should be instigated at 6 months.
On follow-up the incidence of recurrent liver metastases following liver resection in patients whose colorectal cancer was Dukes' B and C was similar (49 vs 50\%). Recurrence after 6 months was higher in patients with Dukes' B cancer, although the difference was not statistically significant. A predominance of late recurrences in the less-advanced Dukes stage may have been anticipated and the lack of significance may be owing to the small number of patients in the study and requires to be validated in a larger series. The Dukes stage of the colon cancer is a factor influencing outcome in multivariate analysis of first liver resection (Fong et al, 1999). In this study, the Dukes stage did not influence the recurrence rate following liver resection. No association was observed between the number of tumour nodules and frequency of recurrence. Although the presence of more than a single metastasis has been reported to be associated with a poorer overall outcome with liver resection (Fong et al, 1999; Muratore et al, 2001; Yamada et al, 2001b), whether it is the absolute number of metastases or the total volume of metastatic disease that affects outcome is not known (Muratore et al, 2001). In the current study, the outcome of solitary and multiple liver metastases in terms of disease recurrence was similar, supporting the role of liver resection in patients with multiple liver metastases. Similar results have been reported in the literature (Ambiru et al, 1999; Fong et al, 1999; Yamamoto et al, 1999b).

The incidence of nonsecretory colorectal cancers is in the order of 20\% (Duffy, 2001; Carpelan-Holmstrom et al, 2002). In this study, 17 of $76(22 \%)$ patients with colorectal liver metastases had normal tumour markers. The mechanism underlying expression of CEA in patients with liver metastases whose primary cancers did not express CEA are unclear. Possible explanations include the degree of differentiation of the primary tumour, effects of transforming growth factor $\alpha$, tumour/host lymphocyte interactions 
causing hepatocyte apoptosis or a loss of hepatic first-pass effect (Duffy, 2001). An important finding in this study is that $60 \%$ of the patients who had normal tumour markers before resection of their liver metastases had elevated tumour markers with recurrence. This would suggest that serial tumour marker assessments should be performed in all patients during follow-up irrespective of the levels before resection of the metastases. While the role of CEA in screening following resection of colorectal liver metastases is welldocumented (Hohenberger et al, 1994; McCall et al, 1994; Paganuzzi et al, 1994; Lucha et al, 1997; Novell et al, 1997; Hocking and Morris, 1998; Wichmann et al, 2000; Ishizuka et al, 2001), there is less published evidence on the use of CA 19-9 (Ishizuka et al, 2001; Carpelan-Holmstrom et al, 2002). In the present study, CEA and CA19-9 were found to be of similar value and complementary in the detection of recurrence following liver resection.

In this study, a protocol of using both tumour markers and CT in the postoperative period improved the detection of recurrent disease. The likelihood of repeat hepatic resection for patients with isolated liver recurrences is less with early recurrences (in this study none) as compared to delayed isolated liver recurrence. This finding needs to be validated in larger studies. Even if early detection does not allow potentially curative resection of recurrent liver metastases, there may be significant clinical benefit to the earlier commencement of systemic chemotherapy or the use of radiofrequency ablation (Elias et al, 2004; Gillams and Lees, 2005). The timing of recurrence and its implications for therapy and patient outcome is vital to the design of follow-up protocols.

Cost-benefit analysis must be considered carefully when designing a follow-up screening protocol. This study would suggest that a similar detection of resectable disease could be achieved at lower cost by a protocol of serial tumour marker estimations and CT scans starting at 6 months after initial surgery. This approach, however, assumes that early recurrences (within 6 months) are never resectable and also disregards the possible benefit from the early commencement of chemotherapy or radiofrequency ablation in patients with unresectable disease. The issue of cost-benefit analysis in this context requires further analysis. However, this altered protocol would significantly decrease the costs from $£ 31008$ to $£ 22542$ per patient potentially cured per year $(P<0.001)$. Similar costs have been reported with an aggressive approach to colorectal liver metastases that have resulted in an increase in quality of life adjusted years (Gazelle et al, 2003).

The design of a follow-up protocol depends on the pattern of recurrent disease. In the present study, recurrence was found in $47 \%$ of patients within 2 years of liver resection for colorectal metastases. The initial 2-year period is recognised as being the main-risk period for recurrence (Scheele et al, 1995). Disease recurrence occurs in the majority of patients in the liver alone and in a proportion of these a further curative resection may be possible. Our data would suggest that tumours recurring early following liver resection are less likely to be amenable to reresection. However, this observation was based on a small number of patients and needs to be validated by data from other centres. The pattern of recurrence would support a surveillance programme being more intense in the first 2 years, but the frequency of imaging within this time frame requires further investigation.

In conclusion, this study has demonstrated that both CEA and CA19-9 are useful in the detection of recurrent disease following potentially curative resection of liver metastases and these are complementary to spiral CT. Early recurrences were not amenable to further surgery, but those detected after 6 months were often resectable. The tumour marker/CT follow-up protocol costs were $£ 31 \mathrm{~K}$ per patient per year.

\section{REFERENCES}

Agrawal N, Fowler AL, Thomas MG (2006) The routine use of intraoperative ultrasound in patients with colorectal cancer improves the detection of hepatic metastases. Colorectal Dis 8: $192-194$

Ambiru S, Miyazaki M, Isono T, Ito H, Nakagawa K, Shimizu H, Kusashio K, Furuya S, Nakajima N (1999) Hepatic resection for colorectal metastases: analysis of prognostic factors. Dis Colon Rectum 42: $632-639$

Ballantyne GH, Quin J (1993) Surgical treatment of liver metastases in patients with colorectal cancer. Cancer 71: 4252-4266

Baulieu F, Bourlier P, Scotto B, Mor C, Eder V, Picon L, De Calan L, Dorval E, Pottier JM, Baulieu JL (2001) The value of immunoscintigraphy in the detection of recurrent colorectal cancer. Nucl Med Commun 22: 12951304

Beard SM, Holmes M, Price C, Majeed AW (2000) Hepatic resection for colorectal liver metastases: a cost-effectiveness analysis. Ann Surg 232: $763-776$

Bengtsson G, Carlsson G, Hafstrom L, Jonsson PE (1981) Natural history of patients with untreated liver metastases from colorectal cancer. Am J Surg 141: $586-589$

Bhattacharjya S, Bhattacharjya T, Baber S, Tibballs JM, Watkinson AF, Davidson BR (2004) Prospective study of contrast-enhanced computed tomography, computed tomography during arterioportography, and magnetic resonance imaging for staging colorectal liver metastases for liver resection. Br J Surg 91: $1361-1369$

Biggs CG, Ballantyne GH (1994) Postoperative follow-up for colorectal cancer: sensitivity vs. cost effectiveness. In Current Opinion in General Surgery Current Science, Daly JM. (ed) 2nd Edition, pp 94-102. Philadelphia

Bolton JS, Fuhrman GM (2000) Survival after resection of multiple bilobar hepatic metastases from colorectal carcinoma. Ann Surg 231: 743-751

Carpelan-Holmstrom M, Louhimo J, Stenman UH, Alfthan H, Haglund C (2002) CEA, CA 19-9 and CA 72-4 improve the diagnostic accuracy in gastrointestinal cancers. Anticancer Res 22: 2311-2316
Daly JM, Bertagnolli M, De Cosse JJ, Morton DJ (1989) Oncology. In Principles of Surgery, Schwartz SI, Shires GT, Spencer FC (eds) pp 297 360. New York: McGraw Hill Book Co

Duffy MJ (2001) Carcinoembryonic antigen as a marker for colorectal cancer: is it clinically useful? Clin Chem 47: 624-630

Elias D, Baton O, Sideris L, Matsuhisa T, Pocard M, Lasser P (2004) Local recurrences after intraoperative radiofrequency ablation of liver metastases: a comparative study with anatomic and wedge resections. Ann Surg Oncol 11: 500-505

Fantini GA, DeCosse JJ (1990) Surveillance strategies after resection of carcinoma of the colon and rectum. Surg Gynecol Obstet 171: 267-273

Fong Y, Fortner J, Sun RL, Brennan MF, Blumgart LH (1999) Clinical score for predicting recurrence after hepatic resection for metastatic colorectal cancer: analysis of 1001 consecutive cases. Ann Surg 230: 309-318

Fujimoto S, Miyazaki M, Kitsukawa Y, Higuchi M, Okui K (1985) Longterm survivors of colorectal cancer with unresectable hepatic metastases. Dis Colon Rectum 28: 588-591

Gazelle GS, Hunink MG, Kuntz KM, McMahon PM, Halpern EF, Beinfeld M, Lester JS, Tanabe KK, Weinstein MC (2003) Cost-effectiveness of hepatic metastasectomy in patients with metastatic colorectal carcinoma: a statetransition Monte Carlo decision analysis. Ann Surg 237: 544-555

Giacchi R, Sebastiani M, Lungarotti F (1988) Natural history of synchronous hepatic metastases from a non-treated colorectal cancer. J Chir (Paris) 125: 419-423

Gillams AR, Lees WR (2005) Radiofrequency ablation of colorectal liver metastases. Abdom Imag 30: 419-426

Glover C, Douse P, Kane P, Karani J, Meire H, Mohammadtaghi S, AllenMersh TG (2002) Accuracy of investigations for asymptomatic colorectal liver metastases. Dis Colon Rectum 45: 476-484

Hocking RA, Morris DL (1998) Patterns of serum CEA fall after hepatic arterial chemotherapy as sole therapy and combined with cryotherapy for colorectal metastases. Aust N Z J Surg 68: 722-724 
Hohenberger P, Schlag PM, Gerneth T, Herfarth C (1994) Pre- and postoperative carcinoembryonic antigen determinations in hepatic resection for colorectal metastases. Predictive value and implications for adjuvant treatment based on multivariate analysis. Ann Surg 219: 135-143

Ishizuka D, Shirai Y, Sakai Y, Hatakeyama K (2001) Colorectal carcinoma liver metastases: clinical significance of preoperative measurement of serum carcinoembryonic antigen and carbohydrate antigen 19-9 levels. Int J Colorectal Dis 16: $32-37$

Leow CK, Lau WY, Li AK (1997) Surgical resection of colorectal hepatic metastases. Hong Kong Med J 3: 50-56

Longo WE, Johnson FE (2002) The preoperative assessment and postoperative surveillance of patients with colon and rectal cancer. Surg Clin N Am 82: $1091-1108$

Lucha Jr PA, Rosen L, Olenwine JA, Reed III JF, Riether RD, Stasik Jr JJ, Khubchandani IT (1997) Value of carcinoembryonic antigen monitoring in curative surgery for recurrent colorectal carcinoma. Dis Colon Rectum 40: $145-149$

Lyass S, Zamir G, Matot I, Goitein D, Eid A, Jurim O (2001) Combined colon and hepatic resection for synchronous colorectal liver metastases. J Surg Oncol 78: 17-21

Machi J, Isomoto H, Yamashita Y, Kurohiji T, Shirouzu K, Kakegawa T (1987) Intraoperative ultrasonography in screening for liver metastases from colorectal cancer: comparative accuracy with traditional procedures. Surgery 101: $678-684$

Malafosse R, Penna C, Sa CA, Nordlinger B (2001) Surgical management of hepatic metastases from colorectal malignancies. Ann Oncol 12: 887 - 894

McCall JL, Black RB, Rich CA, Harvey JR, Baker RA, Watts JM, Toouli J (1994) The value of serum carcinoembryonic antigen in predicting recurrent disease following curative resection of colorectal cancer. Dis Colon Rectum 37: $875-881$

McCormick D, Kibbe PJ, Morgan SW (2002) Colon cancer: prevention, diagnosis, treatment. Gastroenterol Nurs 25: 204-211

Muratore A, Polastri R, Bouzari H, Vergara V, Ferrero A, Capussotti L (2001) Repeat hepatectomy for colorectal liver metastases: a worthwhile operation? J Surg Oncol 76: 127-132

Norum J, Gerner T, Bergan A, Lange O (1997) Follow up after potential curative surgery of colorectal cancer. Guidelines from the Norwegian Gastrointestinal Cancer Group. Tidsskr Nor Laegeforen 117: 2965-2968

Novell F, Trias M, Molina R, Filella X (1997) Detection of occult liver metastases in colorectal cancer by measurement of biliary carcinoembryonic antigen. Anticancer Res 17: 2743-2746

Paganuzzi M, Onetto M, de Paoli M, Castagnola M, de Salvo L, Civalleri D, Grossi CE (1994) Carcinoembryonic antigen (CEA) in serum and bile of colorectal cancer patients with or without detectable liver metastases. Anticancer Res 14: $1409-1412$

Rudy DR, Zdon MJ (2000) Update on colorectal cancer. Am Fam Physician 61: $1759-1770$
Ruers T, Bleichrodt RP (2002) Treatment of liver metastases, an update on the possibilities and results. Eur J Cancer 38: 1023-1033

Ruiz E, Celis J, Berrospi F, Payet E (2000) Surgical treatment of the liver metastases from colorectal cancer. Rev Gastroenterol Peru 20: 376-383

Scheele J, Stang R, Altendorf-Hofmann A, Paul M (1995) Resection of colorectal liver metastases. World J Surg 19: 59-71

Scheele J, Stangl R, Altendorf-Hofmann A (1990) Hepatic metastases from colorectal carcinoma: impact of surgical resection on the natural history. Br J Surg 77: $1241-1246$

Sugihara K, Hojo K, Moriya Y, Yamasaki S, Kosuge T, Takayama T (1993) Pattern of recurrence after hepatic resection for colorectal metastases. $\mathrm{Br}$ J Surg 80: $1032-1035$

Suzuki S, Sakaguchi T, Yokoi Y, Kurachi K, Okamoto K, Okumura T, Tsuchiya Y, Nakamura T, Konno H, Baba S, Nakamura S (2001) Impact of repeat hepatectomy on recurrent colorectal liver metastases. Surgery 129: $421-428$

Tartter PI, Slater G, Gelernt I, Aufses Jr AH (1981) Screening for liver metastases from colorectal cancer with carcinoembryonic antigen and alkaline phosphatase. Ann Surg 193: 357-360

Topal B, Kaufman L, Aerts R, Penninckx F (2003) Patterns of failure following curative resection of colorectal liver metastases. Eur J Surg Oncol 29: $248-253$

Tuttle TM, Curley SA, Roh MS (1997) Repeat hepatic resection as effective treatment of recurrent colorectal liver metastases. Ann Surg Oncol 4: $125-130$

Weber SM, Jarnagin WR, DeMatteo RP, Blumgart LH, Fong Y (2000) Survival after resection of multiple hepatic colorectal metastases. Ann Surg Oncol 7: 643-650

Wichmann MW, Lau-Werner U, Muller C, Hornung HM, Stieber P, Schildberg FW (2000) Carcinoembryonic antigen for the detection of recurrent disease following curative resection of colorectal cancer. Anticancer Res 20: $4953-4955$

Yamada H, Katoh H, Kondo S, Okushiba S, Morikawa T (2001a) Repeat hepatectomy for recurrent hepatic metastases from colorectal cancer. Hepatogastroenterology 48: $828-830$

Yamada H, Kondo S, Okushiba S, Morikawa T, Katoh H (2001b) Analysis of predictive factors for recurrence after hepatectomy for colorectal liver metastases. World J Surg 25: $1129-1133$

Yamaguchi J, Yamamoto M, Komuta K, Fujioka H, Furui JI, Kanematsu T (2000) Hepatic resections for bilobar liver metastases from colorectal cancer. J Hepatobiliary Pancreat Surg 7: 404-409

Yamamoto J, Kosuge T, Shimada K, Yamasaki S, Moriya Y, Sugihara K (1999a) Repeat liver resection for recurrent colorectal liver metastases Am J Surg 178: $275-281$

Yamamoto J, Shimada K, Kosuge T, Yamasaki S, Sakamoto M, Fukuda H (1999b) Factors influencing survival of patients undergoing hepatectomy for colorectal metastases. Br J Surg 86: $332-337$ 